VARIATION IN CONCENTRATION OF FLAVONOIDS AND

CHLOROPHYLL, AND CHANGES ON MORPHOLOGY AND FOLIAR ANATOMY, DUE TO VISIBLE (PAR) OR ULTRAVIOLET (UVA, UVB) RADIATION IN BACCHARIS LATIFOLIA

\section{VARIACIÓN EN LA CONCENTRACIÓN DE FLAVONOIDES Y CLOROFILA, Y CAMBIOS EN LA MORFOLOGÍA Y ANATOMÍA FOLIAR, DEBIDOS A RADIACIÓN VISIBLE (PAR) O ULTRAVIOLETA (UVA, UVB) EN BACCHARIS LATIFOLIA}

Full original article
Received 09132020

Accepted 12142020

Published 12302020

Vol. 37, No.5, pp. 210-222, Nov./Dic.2020

Revista Boliviana de Química

37(5), 210-222, Nov./Dec. 2020

Bolivian Journal of Chemistry

DOI : $10.34098 / 2078-3949.37 .5 .1$

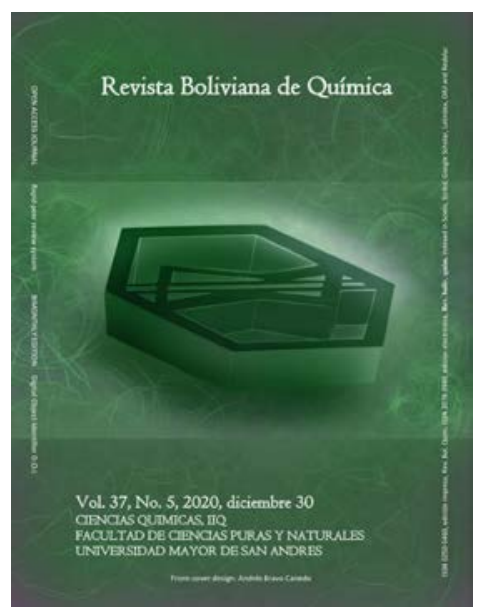

Juan C. Peñaranda ${ }^{1}$, Gloria C. Rodrigo², Armando R. Ticona-Bustillos ${ }^{3}$, Esther Valenzuela C. ${ }^{1}$, Silvia Ramos ${ }^{4}$, Angela San Martin ${ }^{4}$, Flavio G. Ghezzi ${ }^{3}$, Giovanna R. Almanza ${ }^{4, *}$

${ }^{1}$ La Paz Botanical Garden, National Herbarium of Bolivia LPB, Instituto de Ecología IE, Department of Biology, Facultad de Ciencias Puras y Naturales FCPN, Universidad Mayor de San Andrés UMSA, P.O. Box 10077, Calle Andrés Bello s/n, Ciudad Universitaria Cota Cota, phone +59122799459 , La Paz, Bolivia, www.umsa.bo

2Instituto de Biología Molecular y Biotecnología IBMB, Department of Biology, Facultad de Ciencias Puras y Naturales FCPN, Universidad Mayor de San Andrés UMSA, P.O. Box 10077, Calle Andrés Bello s/n, Ciudad Universitaria Cota Cota, phone +59122799459 , La Paz, Bolivia, www.umsa.bo

${ }^{3}$ Instituto de Investigaciones Físicas IIF, Department of Physics, Facultad de Ciencias Puras y Naturales FCPN, Universidad Mayor de San Andrés UMSA, P.O. Box 635, Calle Andrés Bello s/n, Ciudad Universitaria Cota Cota, phone +59122792999 , La Paz, Bolivia, www.umsa. bo

${ }^{4}$ Laboratory of Bioorganic, Instituto de Investigaciones Químicas IIQ, Chemical Sciences Department, Facultad de Ciencias Puras y Naturales FCPN, Universidad Mayor de San Andrés UMSA, P.O. Box 303, Calle Andrés Bello s/n, Ciudad Universitaria Cota Cota, phone +59122792238, La Paz, Bolivia, www.iiq.umsa.bo

Keywords: Baccharis latifolia, UV, PAR, Total flavonoids, Chlorophyll.

Palabras clave: Baccharis latifolia, UV, PAR, Flavonoides totales, Clorofila.

\title{
ABSTRACT
}

Baccharis latifolia is a plant of medicinal and industrial interest with an abundance of flavonoids within its leaves. The present study focused on identifying the changes in total flavonoids, chlorophyll concentration, plant growth, and leaf anatomy at different wavelengths (UVA, UVB and PAR) under controlled conditions. Statistical analyzes were carried

$\begin{aligned} & \text { Downloadable from: Revista Boliviana de } \\ & \text { http://www.bolivianchemistryjournal.org, }\end{aligned}$
$\underline{\underline{y}}$




\section{Juan C. Peñaranda et al. RBQ Vol.37, No.5, pp. 210-222, 2020}

out using nonparametric Kruskal-Wallis and post hoc Bonferroni tests. The results suggest that favorable conditions for the production of flavonoids occur when exposed to UVB50 treatment (50\% UVB with respect to daily solar radiation), while it is observed that low levels of PAR play an important role in reducing the flavonoids. The chlorophyll concentration is favored by PAR and UVA50 treatments; however, it is negatively influenced by UVB100 and UVB150. Vegetative growth does not show strong differences when comparing PAR treatments, however, it does present better results in relation to treatments that include UVA or UVB radiation. The foliar anatomy shows strong differences between the treatments and is favored mainly by the UVA100 and UVA150 treatments, while it is negatively affected by the UVB100 and UVB150. The observed results show that Baccharis latifolia is a plastic species that can accommodate various levels of light and wavelength, however, it presents differentiated responses in growth, physiology and foliar anatomy that affect the concentration of compounds.

\section{*Correspondent author: galmanza@fcpn.edu.bo}

\section{RESUMEN}

Baccharis latifolia es una planta de interés medicinal e industrial con presencia importante de flavonoides en las hojas. El presente estudio se centró en identificar los cambios en flavonoides totales, concentración de clorofilas, crecimiento de la planta y anatomía foliar bajo diferentes longitudes de onda (UVA, UVB y PAR) en condiciones controladas. Se realizaron análisis estadísticos utilizando pruebas no paramétricas Kruskal-Wallis y post hoc Bonferroni. Los resultados sugieren que mejores condiciones para la generación de flavonoides se dan bajo el tratamiento UVB50 (50\% de UVB respecto a la radiación solar diaria), además, se observa que bajos niveles de PAR juegan un papel importante en la reducción de los mismos. La concentración de clorofilas se ve favorecida por los tratamientos de PAR y UVA50, sin embargo, es negativamente influenciada por los tratamientos UVB100 y UVB150. El crecimiento vegetativo no muestra fuertes diferencias comparando los tratamientos de PAR, sin embargo, si presenta mejores resultados en relación a tratamientos que adicionen radiación UVA o UVB. La anatomía foliar muestra fuertes diferencias entre los tratamientos y se ve favorecida principalmente por los tratamientos UVA100 y UVA150, en cambio es negativamente afectada por los tratamientos UVB100 y UVB150. En relación a nuestros resultados se observa que Baccharis latifolia es una especie plástica que puede acomodarse a varios niveles de luz y longitud de onda, sin embargo, presenta respuestas diferenciadas a nivel de su crecimiento, fisiología y anatomía foliar que repercuten en la concentración de compuestos fenólicos, clorofilas y crecimiento vegetativo.

\section{INTRODUCCIÓN}

Baccharis latifolia (Ruiz \& Pav.) Pers. (Asteraceae) es un arbusto perenne de valles secos interandinos muy utilizado en medicina tradicional [1]. Esta especie contiene varios compuestos bioactivos, entre ellos flavonoides, que son responsables de parte de su actividad antiinflamatoria y de su utilización en la industria farmacéutica [2-3]. Existen reportes que apuntan a una mayor concentración de flavonoides en época húmeda y se argumenta alguna relación con características meteorológicas, suelo y radiación solar [2-4].

Luz, agua y temperatura son los principales factores abióticos para el crecimiento de las plantas [5], de estos tres factores la luz es fundamental para el crecimiento y normal desenvolvimiento de todas las funciones biológicas [6-9] debido a que se relaciona directamente con fisiología, bioquímica y procesos morfológicos [10-11].

A nivel del metabolismo secundario una respuesta comúnmente reportada a la radiación ultravioleta (UV) es el aumento en la concentración de compuestos fenólicos, que se depositan en vacuolas de las células epidérmicas [1213]. Además, la medición de clorofilas y carotenoides han demostrado ser útiles indicadores para evaluar la tolerancia a UV [14] esto debido a que la clorofila desempeña un papel crucial en la producción de foto-asimilados que son posteriormente utilizados para la construcción de metabolitos secundarios y el crecimiento de la planta [5].

También, la estructura de la hoja influye ampliamente en la capacidad de protección UV, observándose en algunos casos un cambio en el grosor y, en otros casos, formación de apéndices [15].

Estudios para evaluar el efecto del espectro desde el ultravioleta hasta el infrarrojo se han realizado en varias especies de plantas con diferentes fines (producción, determinación de rutas metabólicas, historia natural), la mayoría en cultivos y especies con potencial comercial. Existen respuestas morfo-fisiológicas similares en especies aclimatadas a sol directo con alta concentración de ultravioleta en contraparte a las que viven en sombra con mayor enriquecimiento de rojo [16].

Considerando que Baccharis latifolia es una especie económicamente importante, investigar los efectos que tiene la radiación ultravioleta en condiciones controladas sobre la concentración de flavonoides y clorofilas puede brindar pautas en la colecta de hojas y producción en campo. En la presente investigación se propuso evaluar la aclimatación

$\begin{array}{ccc}\begin{array}{c}\text { Downloadable from: Revista Boliviana de } \\ \text { http://www.bolivianchemistryjournal.org, }\end{array} & 211 \quad \begin{array}{c}\text { Química. Volumen } 37 \text { N 5. Año } 2020 \\ \text { http://www.scribd.com/bolivianjournalofchemistry }\end{array}\end{array}$


Juan C. Peñaranda et al. RBQ Vol.37, No.5, pp. 210-222, 2020

de plantas de Baccharis latifolia bajo diferentes tratamientos de aclimatación y posteriormente inducción a estrés UV, cuantificando el crecimiento vegetativo la producción de flavonoides, clorofila y cambios en la morfo-anatomía foliar.

\section{EXPERIMENTAL}

\section{Material biológico}

Los individuos de Baccharis latifolia fueron germinados en cajas Petri a $9.25 \mu \mathrm{mol} / \mathrm{m} 2 \mathrm{~s}$ de densidad de flujo fotónico (PPFD) y 2ml de agua destilada, posteriormente fueron trasplantados en sustrato estándar con arena, turba y arcilla en relación 40:40:30 respectivamente y bajo una concentración de $92.5 \mu \mathrm{mol} / \mathrm{m} 2 \mathrm{~s}$ (PPFD). De un stock de 50 individuos se escogieron 28 individuos para la primera fase (7 por tratamiento) y de un segundo stock 100 individuos se escogieron 49 (7 por tratamiento). Los individuos fueron distribuidos según características similares (altura y número de hojas) para los diferentes tratamientos.

\section{Tratamientos de irradiación}

Los tratamientos de irradiación se dividieron en dos etapas, la aclimatación de plantas (Fase 1) y posteriormente la inducción al estrés ultravioleta (Fase 2). Las lámparas utilizadas fueron TL-D 36W/54-765 1SL (400-700 nm) para el PAR (Radiación Fotosintéticamente Activa), TL-K 40W/10-R UVA (315- 380nm) para UVA y TL 40W/12 RS SLV/25 $(290$ - 315nm) para UVB todas de la marca PHILIPS. La caracterización se realizó con dosímetro GigahertzOptick XD-9501 y medidor PAR LGBQM Hydrofarm.

Tabla 1. Tratamientos fase 1, valores de densidad de flujo de fotones por tratamiento (PPFD), fotoperiodo, número de lámparas utilizadas y porcentaje de radiación de cada tratamiento respecto a días nublados en época húmeda.

\begin{tabular}{c|cccc} 
TRATAMIENTO & CARACTERÍstICAS & $\begin{array}{c}\text { FOTOPERIODO } \\
\text { HORAS/DÍA }\end{array}$ & $\boldsymbol{\mu m o l} / \mathbf{m}^{2} \mathbf{s}^{2}$ & \% RESPECTO AL SOL (DIFUSA) \\
\hline 130 PPFD & 8 lámparas blanca & 8 & 130 & 60 \\
265 PPFD & 8 lámparas blanca & 12 & 265 & 75 \\
400 PPFD & 10 lámparas blanca & 12 & 400 & 80 \\
533 PPFD & 10 lámparas blanca & 16 & 533 & 110 \\
\hline
\end{tabular}

Tabla 2. Tratamientos fase 2, valores de tipo de UV utilizada (UVA, UVB y Blanca), intensidad del tratamiento (Watt $/ \mathrm{m}^{2} \mathrm{~s}$ ), número de lámparas y porcentaje de radiación acumulada respecto al sol.

\begin{tabular}{|c|c|c|c|c|}
\hline TRATAMIENTO & CARACTERÍSTICAS & $\begin{array}{l}\text { FOTOPERIODO } \\
\text { POR DÍA }\end{array}$ & WATT/M2S & $\begin{array}{c}\% \text { RESPECTO } \\
\text { AL SOL }\end{array}$ \\
\hline UVA50 & 6 lámparas UVA / 8 lámparas blanca & $12 / 12$ & 0.931 & 50 \\
\hline UVA100 & 4 lámparas UVA / 8 lámparas blanca & $12 / 12$ & 1.976 & 100 \\
\hline UVA150 & 2 lámparas UVA / 8 lámparas blanca & $12 / 12$ & 2.928 & 150 \\
\hline UVB50 & 2 lámparas UVB / 8 lámparas blanca & $2 / 12$ & 2.912 & 50 \\
\hline UVB100 & 2 lámparas UVB / 8 lámparas blanca & $4 / 12$ & 2.912 & 100 \\
\hline UVB150 & 2 lámparas UVB / 8 lámparas blanca & $6 / 12$ & 2.912 & 150 \\
\hline LUZ BLANCA & 8 lámparas blanca & 12 & 0.01 & 75 \\
\hline
\end{tabular}

Para la experimentación de la fase 1 se armaron cuatro tratamientos de luz blanca donde se varió el número de lámparas y las horas luz con el fin de simular horas acumuladas de radiación solar (tabla 1). El experimento tuvo una duración de tres meses y se detuvo al observar reducción en la vitalidad de las plantas.

Una vez terminado el proceso de aclimatación y determinada la mejor concentración de luz blanca para el desarrollo de las plantas, se procedió a instalar la fase 2, con tres tratamientos por cada radiación ultravioleta (UVA y UVB) y un tratamiento control con luz blanca derivada del tratamiento 265 PPFD del anterior ensayo (tabla 2). La experimentación en esta fase duro dos meses y se detuvo al observar perdida de vitalidad en el tratamiento UVB150.

Ambas experimentaciones contaron con 7 individuos por tratamiento. El régimen de riego, temperatura, humedad, $\mathrm{pH}$ de suelo, conductividad suelo y control de plagas fue similar para todos los tratamientos.

\section{Crecimiento de las plantas}

\begin{tabular}{|c|c|c|}
\hline $\begin{array}{l}\text { Downloadable from: Revista Boliviana de } \\
\text { http://www.bolivianchemistryjournal.org, }\end{array}$ & 212 & $\begin{array}{l}\text { Química. Volumen } 37 \text { No5. Año } 2020 \\
\text { http://www.scribd.com/bolivianjournalofchemistry }\end{array}$ \\
\hline
\end{tabular}


Juan C. Peñaranda et al. RBQ Vol.37, No.5, pp. 210-222, 2020

En la fase 1 se realizó la medición del número de hojas y la altura del tallo cada tres semanas conjuntamente con la extracción de material foliar para la cuantificación química y para los estudios histológicos. En la fase 2 solamente se cuantificó la altura y el número de hojas inicial y final con el fin de no mover las plantas dentro del tratamiento. Además, se cuantificó la biomasa de cada planta extrayendo los tallos y hojas y secándolos a 500C para su posterior pesaje.

\section{Área foliar}

Para la fase 2 se realizó la medición del área foliar de 3 hojas por planta, se procedió a sacar una fotografía con escala y posteriormente el análisis de imagen con imagen $J$.

\section{Morfo anatomía foliar}

Para ambas fases se extrajeron tres hojas por planta, de cada hoja se realizó un corte transversal en posición media. Los cortes se realizaron con ayuda de micrótomo semiautomático y para endurecer la lámina foliar se utilizaron tratamientos de deshidratación con diferentes concentraciones de alcohol. Los cortes se montaron en porta objetos con bálsamo de Canadá para preservar la estructura y fueron medidos en microscopio a escala. Para obtener la relación PE/PL se realizó la división del parénquima en empalizada (PE) entre el parénquima lagunar (PL).

\section{Concentración de clorofilas $(\mathrm{Ch})$}

La extracción de clorofila se realizó a partir de $0.5 \mathrm{~g}$ de hoja, triturada en mortero con ayuda de nitrógeno líquido. Se extrajeron los pigmentos con $10 \mathrm{ml}$ de etanol de 960 y se centrifugó a $10000 \mathrm{rpm}$ por 15 minutos, se recuperó $0.5 \mathrm{ml}$ del sobrenadante y se aforó a $5 \mathrm{ml}$ con etanol al $96 \%$. Posteriormente se procedió a la lectura en espectrofotómetro visible a 646 y $663 \mathrm{~nm}$, en muestras por triplicado. Para realizar el cálculo de clorofila A (ChA) y clorofila B (ChB) se utilizaron las ecuaciones de Sumanta [17].

\section{Obtención de extractos para cuantificación de flavonoides}

Las hojas colectadas fueron secadas a temperatura ambiente, trituradas de forma manual y depositadas en frascos para la obtención de extractos. La extracción se realizó por maceración con etanol de $96^{\circ}$, previamente destilado, durante 15 min a temperatura ambiente. Los extractos fueron filtrados y concentrados hasta sequedad por rotaevaporación para ser guardados a temperatura ambiente en lugares secos y oscuros hasta su uso [18].

\section{Cuantificación de flavonoides totales}

Para la cuantificación de flavonoides totales en extractos se utilizó el método colorimétrico por quelación con AlCl3 [19]. Como flavonoide estándar se utilizó la Luteolina [20]. Se colocaron $0.5 \mathrm{ml}$ de muestra con $1.5 \mathrm{ml}$ de etanol destilado $96^{\circ}$, a los cuales se adicionó $2.8 \mathrm{ml}$ de agua destilada, $0.1 \mathrm{ml}$ de $10 \%$ de solución de $\mathrm{AlCl} 3(\mathrm{~m} / \mathrm{v})$, y $0.1 \mathrm{ml}$ de $1 \mathrm{~mol} / \mathrm{L}$ de solución de acetato de potasio, causando la formación de un complejo amarillo intenso. La solución se incubó durante 30 a 40 min y finalmente se realizó un análisis espectral a 406 nm [21].

\section{Análisis estadístico}

Los datos de las variables analizadas fueron sometidos a un análisis de homogeneidad de varianzas Levene donde algunas de las variables mostraron significancia motivo por el cual se escogió el análisis de varianza no paramétrico Kruskal-Wallis. Los análisis post hoc consintieron en pruebas Bonferroni, donde se compararon las medias de las variables que mostraron diferencias significativas. La unidad experimental para cada una de las variables estuvo conformada por cada planta, con 7 repeticiones. Para el análisis de cada una de las variables, los datos promedios de las unidades muéstrales se evaluaron mediante SPSS STATICIS 25 con un nivel de significancia de 0.05 .

\section{RESULTADOS Y DISCUSIÓN}

\section{Fase 1 Condiciones de aclimatación}

Las plantas de Baccharis latifolia responden de manera diferente en relación a la elongación del tallo, los valores más bajos a las 12 semanas se observan a 400 PPFD $(0.95 \mathrm{~cm})$ y los más altos a 533 PPPFD $(2.45 \mathrm{~cm})$ (tabla 3$)$. El número de hojas presentó los valores más altos a 130 PPFD (41 hojas) y los más bajos a 265 PPFD (19 hojas) (tabla 3). Esto muestra que bajas concentraciones de luz blanca disminuyen el crecimiento del tallo e incrementan el número de hojas. Respecto a la morfo anatomía foliar se observa una relación menor a 1 entre el PE/PL, siendo la más cercana el

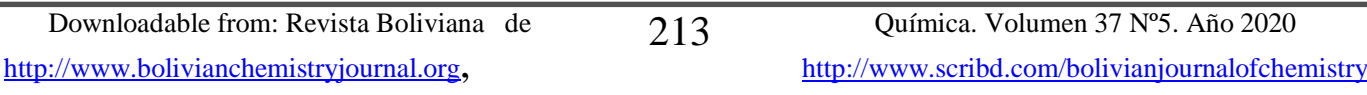


Juan C. Peñaranda et al. RBQ Vol.37, No.5, pp. 210-222, 2020

tratamiento 533 PPFD con 0.90 (tabla 3). Los análisis estadísticos no mostraron diferencias significativas para las variables de crecimiento ni para las variables de anatomía foliar (tabla 4).

Otros estudios [22] mostraron que la detención en la iniciación de la hoja bajo oscuridad podría ser debida a la falta de energía, pero también es posible que la luz actúe como una señal ambiental de la iniciación de la hoja, motivo por el cual bajas concentraciones de luz no promuevan el desarrollo apical. Por otro lado, las respuestas a bajos niveles de luz en relación al número de hojas son de dos tipos. A) Mayor aparición de hojas [23-24] y/o B) Aumento del área foliar, para compensar las tasas fotosintéticas por unidad de área [25]. Respecto al cambio en la anatomía foliar, la reducción de la relación PE/PL en los tratamientos de baja intensidad lumínica, se debe a que las células del PL son más eficientes en la captación de luz difusa, por ende, la relación $\mathrm{PE} / \mathrm{PL}<1$ es ventajosa en ambientes de baja concentración de luz como un mecanismo de defensa [16].

Tabla 3. Valores de los promedios y desviación estándar de las variables, altura de la planta, número de hojas y relación parénquima en empalizada / parénquima lagunar (PE/PL) de los tratamientos 133 - 533 PPFD a las 12 semanas.

\begin{tabular}{c|ccc} 
TRATAMIENTOS & ALTURA (CM) & NUM. HOJAS & PE/PL \\
\hline 130 PPFD & $1.75+/-0.3$ & $41+/-5$ & $0.88+/-0.03$ \\
265 PPFD & $2.2+/-0.2$ & $19+/-4$ & $0.81+/-0.02$ \\
400 PPFD & $0.95+/-0.15$ & $32+/-5$ & $0.89+/-0.05$ \\
533 PPFD & $2.45+/-0.3$ & $21+/-5$ & $0.90+/-0.07$ \\
\hline
\end{tabular}
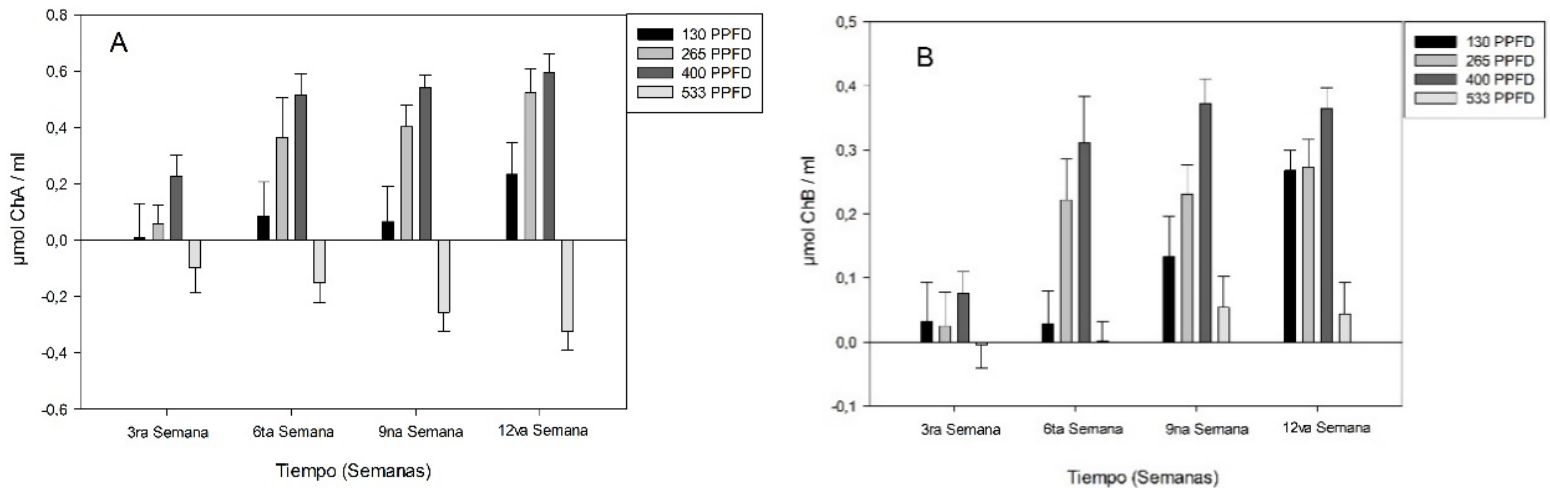

Figura 1. Concentración de ChA (A) y ChB (B) por extracción etanólica cada tres semanas de los tratamientos 130 a 533 PPFD. La concentración se calculó de la resta de los parámetros final vs inicial de cada muestreo.

La concentración de clorofilas presenta un cambio significativo (tabla 4) en relación a los tratamientos planteados (ChA H = 20.36, sig $=<0.01$; $\mathrm{ChB} \mathrm{H}=18.87$, sig $=<0.01$ y ChA/ChB H = 8.14, sig = 0.04). El tratamiento a 400 PPFD exhibió el mayor incremento en concentración de ChA $=1.78 \mu \mathrm{mol} / \mathrm{ml}$ y ChB $=1.09 \mu \mathrm{mol} / \mathrm{ml}$, en cambio el tratamiento a 533 PPFD mostro los valores más bajos de ChA $=-0.97 \mu \mathrm{mol} / \mathrm{ml}$ y ChB $=0.13 \mu \mathrm{mol} / \mathrm{ml}$, los valores negativos se presentan debido a que se utilizó la diferencia entre el valor final y el valor inicial (figura 1). Las pruebas post hoc muestran que el tratamiento más diferenciado es el de 533 PPFD (tabla 5), resultado que también se puede observar en la figura 1, donde la concentración de ChA disminuye a medida que trascurre el tiempo de experimentación, la concentración de ChB disminuye hasta la tercera semana. Este comportamiento se puede deber al estrés fotoxidativo [25-27], limitando la fotosíntesis por la foto inhibición. Altos niveles de luz derivaran a corto plazo en foto inhibición breve y a largo plazo en estrés oxidativo y destrucción del aparato fotosintético. Por otro lado, concentraciones altas de clorofila B en plantas sometidas a condiciones de baja radiación son un mecanismo de adaptación para permitir mayor eficiencia de absorción con menor intensidad lumínica asegurando la tasa fotosintética [28] y la transferencia de hojas de sombra a sol disminuyen el contenido de Chl A por el aumento de la densidad lumínica [29] como se observa entre los tratamientos de 130 a 533 PPFD.

Respecto a la concentración de flavonoides no se presentó diferencias significativas al finalizar la experimentación, los tratamientos 130, 265, 400 y 533 PPFD mostraron un decremento de la concentración respecto al tiempo de experimentación, únicamente el tratamiento 533 PPFD mostro un leve incremento en la concentración de flavonoides (1.3mg/g) hasta la sexta semana (figura 2), este incremento puede ser producido como un mecanismo de defensa para asegurar la funcionalidad en las hojas, por el contrario, bajas concentraciones de luz disminuyen la concentración de flavonoides [30].

$\begin{array}{ccc}\begin{array}{c}\text { Downloadable from: Revista Boliviana de } \\ \text { http://www.bolivianchemistryjournal.org, }\end{array} & 214 & \begin{array}{c}\text { Química. Volumen } 37 \text { Nº5. Año } 2020 \\ \text { http://www.scribd.com/bolivianjournalofchemistry }\end{array}\end{array}$


Analizando la última semana de experimentación (figura 3) se observa que la concentración de luz tiene relación directa con la concentración de flavonoides de manera lineal $\mathrm{Y}=0.00488 \mathrm{x}$ - 17.99 (R2 de 0.91), donde existiría menor concentración de flavonoides a menor concentración de Luz. Estos resultados son comparables con otros estudios donde se indica que la producción de flavonoides es favorecida por altos niveles de radiación solar y disminuyen si no existe el estímulo adecuado [31-35].

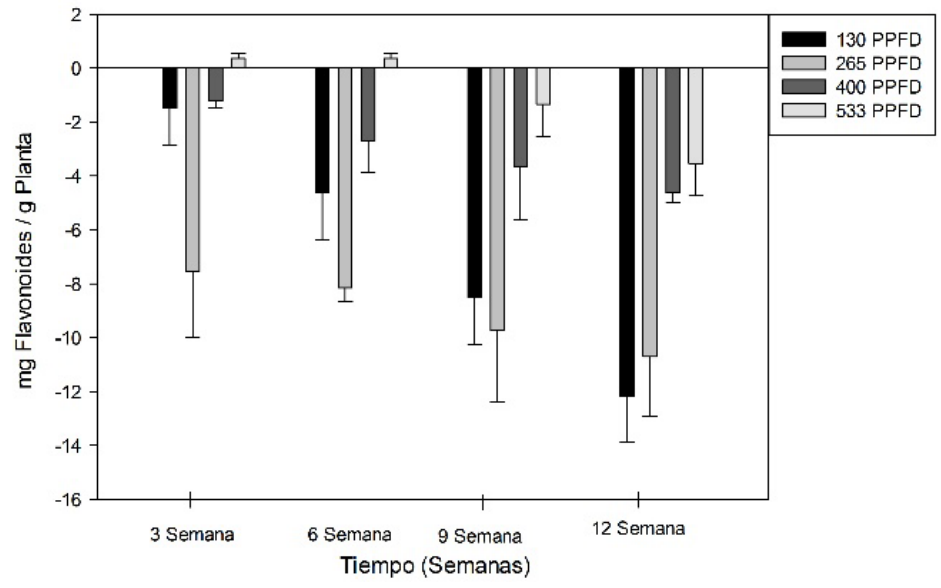

Figura 2. Concentración de flavonoides totales por extracción etanólica en relación a los tratamientos comparados y el tiempo de exposición. Los datos se calcularon a partir de la resta concentración final vs concentración inicial de cada muestreo.

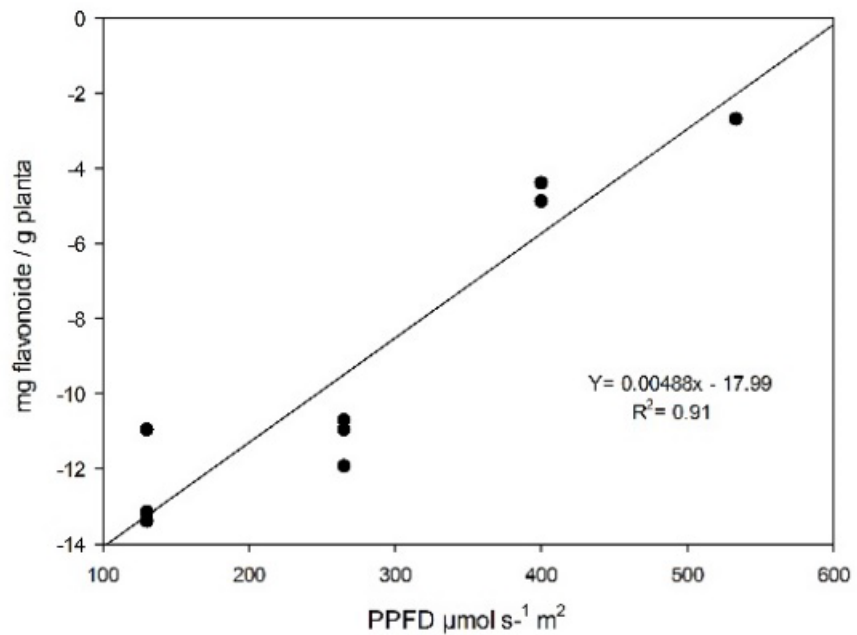

Figura 3. Dispersión de datos de la concentración de flavonoides respecto a los tratamientos de luz. Los datos se calcularon a partir de la resta concentración final vs concentración inicial a las 12 semanas de experimentación.

Tabla 4. Prueba Kruskal - Wallis en relación a las variables de respuesta fase 1, se observa diferencias significativas al 0.05 de confianza en las variables ChA (<0.01), ChB $(<0.01)$ y $\operatorname{ChA} / \mathrm{ChB}(0.04)$.

\begin{tabular}{l|lllr}
\multicolumn{1}{c}{ RESPUESTAS } & \multicolumn{1}{c}{ VARIABLE } & \multicolumn{1}{c}{ H } & GL & SIG. ASINTÓTICA \\
\hline QUíMICAS & Flavonoides totales & 5.97 & 3 & 0.11 \\
& ChA & 20.36 & 3 & $<0.01^{*}$ \\
& ChB & 18.87 & 3 & $<0.01^{*}$ \\
\multirow{2}{*}{ MORFOLÓGICAS } & ChA/ChB & 8.14 & 3 & $0.04^{*}$ \\
& Altura del tallo & 7.54 & 3 & 0.06 \\
& Número de hojas & 3.82 & 3 & 0.28 \\
ANATÓMICAS & PE/PL & 2.87 & 3 & 0.41 \\
\hline
\end{tabular}

Downloadable from: Revista Boliviana de http://www.bolivianchemistryjournal.org,
215

Química. Volumen 37 N5. Año 2020 http://www.scribd.com/bolivianjournalofchemistry 
Tabla 5. Pruebas post-hoc Bonferroni en relación a las variables significativas de la fase 1 la 0.05 de nivel de confianza, se observa diferencias de la clorofila A entre los tratamientos 265 y 400 con el tratamiento 533, además la clorofila B muestra diferencias significativas entre 130, 265, 400 y el tratamiento 533. Respecto a la ChA/ChB se observa diferencias significativas únicamente entre el tratamiento 130 y 265.

\begin{tabular}{r|ccc} 
VARIABLE & TRATAMIENTOS & $\begin{array}{c}\text { ESTADÍSTICO DE } \\
\text { CONTRASTE }\end{array}$ & P. VALOR \\
\hline ChA & $265-533$ & 17.76 & $<0.01^{*}$ \\
ChA & $400-533$ & 20.37 & $<0.01^{*}$ \\
ChB & $130-533$ & 13.87 & $<0.01^{*}$ \\
ChB & $265-533$ & 14.21 & $<0.01^{*}$ \\
ChB & $400-533$ & 21 & $<0.01^{*}$ \\
ChA/ChB & $130-265$ & -11 & $0.02^{*}$ \\
\hline
\end{tabular}

\section{Fase 2 Exposición a estrés ultravioleta}

Los resultados muestran que existe un cambio en el crecimiento de la planta respecto a la radiación ultravioleta. El número de hojas muestra diferencias significativas $(\mathrm{H}=16.94$, sig. $=<0.01)$ respecto al control luz blanca, siendo el tratamiento UVA50 el que presenta la mayor variación según las pruebas post hoc (tabla 6, 8 y 9). El crecimiento del tallo también muestra diferencias significativas respecto al control $(\mathrm{H}=41.22$, sig. $=<0.01)$, siendo el UVA50 el más cercano al control (44 y $41.86 \mathrm{~cm}$ respectivamente) (tabla 6 y 8). Los análisis post hoc muestran que el tratamiento UVB50 es el que presenta los valores más bajos $(24 \mathrm{~cm})$, seguido de UVA100 (28.42 cm) y UVB $150(25.14 \mathrm{~cm})$ (tabla 6, 8 y 9). En relación a la biomasa la UVB 50 y la UVA100 muestran valores similares al control (8.03, 7.24 y $7.81 \mathrm{Kg}$ respectivamente) y todos los demás tratamientos muestran una reducción (tabla 6), sin embargo, las diferencias no son significativas (Tabla 8). El índice de área foliar no presenta diferencias significativas (tabla 8), los valores más bajos se presentan en el tratamiento UVB100 (36.31) y UVB50 (38.92) respecto al control (45.23) (tabla 6). Respuestas en el crecimiento y rasgos morfológicos de las hojas como la reducción del área foliar por UVB podrían ser explicados por la inhibición de la división celular, y el UVA por el aumento del mesófilo (tabla 7) [36]. Además, dosis bajas de radiación UVB y blanca pueden incrementar el crecimiento vegetativo en relación a la biomasa fresca y seca, si bien la UVB puede afectar negativamente el crecimiento de la planta bajas concentraciones pueden promover respuestas de defensa [37].

La anatomía foliar muestra diferencias significativas en todas las variables de respuesta (Mesófilo $\mathrm{H}=37.47$, sig. $=<0.01$; E. adaxial $\mathrm{H}=26.02$, sig. $=<0.01$; E. abaxial $\mathrm{H}=22.63$ sig. $=<0.01 ; \mathrm{PE} \mathrm{H}=36.43$, sig. $=<0.01$; $\mathrm{PL} \mathrm{H}=$ 31.53, sig. $=<0.01$ ) excepto la relación PE/PL (tabla 8). Se observa que la UVA150 y UVA100 favorecen al desarrollo de la hoja y sus tejidos (tabla 7 y 9) mostrando un aumento proporcional en el mesófilo (0.51 y $0.42 \mu \mathrm{m})$, E. adaxial $(0.03$ y $0.03 \mu \mathrm{m})$, E. abaxial $(0.03$ y $0.03 \mu \mathrm{m})$, PE $(0.19$ y $0.17 \mu \mathrm{m})$ y PL $(0.25$ y $0.18 \mu \mathrm{m})$ en relación a el control luz blanca (mesófilo $=0.36 \mu \mathrm{m}, \mathrm{E}$. adaxial $=0.02 \mu \mathrm{m}, \mathrm{E}$ abaxial $=0.02 \mu \mathrm{m}, \mathrm{PE}=0.14 \mu \mathrm{m}$ y PL $=0.17 \mu \mathrm{m})(\mathrm{tabla} 7 \mathrm{y} 9)$. Los valores más bajos se observan en los tratamientos UVB150 y UVB100 (Mesófilo = 0.18 y $0.25 \mu \mathrm{m}$, E. adaxial = 0.01 y $0.01 \mu \mathrm{m}$, E. abaxial $=0.01$ y $0.01 \mu \mathrm{m}, \mathrm{PE}=0.07-\mathrm{y} 0.01 \mu \mathrm{m}, \mathrm{PL}=0.09$ y $0.019 \mu \mathrm{m})$ mostrando que el UVB afecta a la formación de tejidos (tabla 7 y 9).

Tabla 6. Valores de los promedios y desviación estándar de las variables de crecimiento, Biomasa, Índice de Área foliar altura de planta y número de hojas de los tratamientos UVA, UVB y control blanco a las 8 semanas.

\begin{tabular}{|c|c|c|c|c|}
\hline TRATAMIENTO & BIOMASA (KG) & $\begin{array}{c}\text { ÍNDICE DE ÁREA } \\
\text { FOLIAR }\end{array}$ & ALTURA (CM) & NÚM. HOJAS \\
\hline Blanca & $7.81+/-2.05$ & $45.23+/-2.67$ & $44.00+/-7.37$ & $90+/-31.68$ \\
\hline UVA150 & $4.78+/-2.21$ & $42.04+/-5.31$ & $33.57+/-4.92$ & $54+/-11.21$ \\
\hline UVA100 & $7.24+/-2.98$ & $40.54+/-3.00$ & $28.42+/-8.17$ & $56+/-7.56$ \\
\hline UVA50 & $6.37+/-3.18$ & $42.85+/-2.52$ & $41.86+/-11.36$ & $58+/-16.86$ \\
\hline UVB150 & $4.92+/-2.69$ & $42.83+/-2.52$ & $25.28+/-2.23$ & $57+/-18.93$ \\
\hline UVB100 & $5.13+/-2.74$ & $36.31+/-8.53$ & $25.14+/-5.54$ & $60+/-21.83$ \\
\hline UVB50 & $8.03+/-2.72$ & $38.2+/-15.22$ & $24.00+/-4.31$ & $56+/-19.96$ \\
\hline
\end{tabular}

Downloadable from: Revista Boliviana de http://www.bolivianchemistryjournal.org,
216

Química. Volumen 37 N5. Año 2020

http://www.scribd.com/bolivianjournalofchemistry 
Juan C. Peñaranda et al. RBQ Vol.37, No.5, pp. 210-222, 2020

Tabla 7. Valores de los promedios y desviación estándar de las variables de anatomía foliar, ancho de mesófilo, ancho de epidermis adaxial, ancho de epidermis abaxial, parénquima en empalizada (PE), parénquima lagunar (PL) y relación parénquima en empalizada / parénquima lagunar (PE/PL) de los tratamientos UVA, UVB y control blanco a las 8 semanas.

\begin{tabular}{|c|c|c|c|c|c|c|}
\hline & $\begin{array}{l}\text { MESÓFILO } \\
(\mu \mathrm{M})\end{array}$ & $\begin{array}{l}\text { E. ADAXIAL } \\
(\mu \mathrm{M})\end{array}$ & $\begin{array}{c}\text { E. ABAXIAL } \\
(\mu \mathrm{M})\end{array}$ & PE $(\mu \mathrm{M})$ & $\mathrm{PL}(\mu \mathrm{M})$ & $\mathrm{PE} / \mathrm{PL}$ \\
\hline UVB150 & $0.18+/-0.04$ & $0.01+/-0.004$ & $0.01+/-0.005$ & $0.07+/-0.01$ & $0.09+/-0.02$ & $0.79+/-0.1$ \\
\hline UVB100 & $0.25+/-0.02$ & $0.01+/-0.009$ & $0.01+/-0.007$ & $0.01+/-0.01$ & $0.12+/-0.01$ & $0.83+/-0.1$ \\
\hline UVB50 & $0.32+/-0.01$ & $0.01+/-0.001$ & $0.02+/-0.005$ & $0.12+/-0.01$ & $0.15+/-0.01$ & $0.76+/-0.08$ \\
\hline UVA50 & $0.35+/-0.01$ & $0.01+/-0.007$ & $0.02+/-0.004$ & $0.12+/-0.008$ & $0.15+/-0.02$ & $0.93+/-0.1$ \\
\hline UVA100 & $0.42+/-0.02$ & $0.03+/-0.005$ & $0.03+/-0.004$ & $0.17+/-0.02$ & $0.18+/-0.02$ & $0.98+/-0.2$ \\
\hline UVA150 & $0.51+/-0.02$ & $0.03+/-0.007$ & $0.03+/-0.008$ & $0.19+/-0.02$ & $0.25+/-0.02$ & $0.81+/-0.2$ \\
\hline BLANCA & $0.36+/-0.1$ & $0.02+/-0.009$ & $0.02+/-0.008$ & $0.14+/-0.04$ & $0.17+/-0.05$ & $0.86+/-0.1$ \\
\hline
\end{tabular}

La plasticidad fenotípica observada en la respuesta a estrés ultravioleta por parte de Baccharis latifolia es consistente con algunas respuestas de otras especies a esta longitud de onda [38]. El ancho de mesófilo en varios genotipos de plantas, también aumenta cuando se somete a alta intensidad de luz, inducido principalmente por el mayor engrosamiento PE y PL, debido a la mayor elongación celular, ya que el número de capas en estos tejidos permanecen constantes [38]. Por otro lado, el aumento en el grosor de la lámina foliar puede disminuir los efectos foto oxidativos, aumentando el área de disipación de energía, efecto que es promovido por la UVA [39]. Sin embargo, la radiación UVB y su concentración puede inducir negativamente en la diferenciación foliar y adelantar procesos de senescencia a través de la modificación de la estructura de la hoja [40-41].

Los resultados para la concentración de clorofilas muestran diferencias significativas en UVA y UVB respecto al tratamiento control (ChA H $=29.75$, sig. $=<0.01$ y ChB $=27.33$, sig. $=<0.01$ ) (tabla 8). El tratamiento en el que se observa mayor concentración de ChA y ChB es el UVA50 (5.95 umol/ml ChA y $2.13 \mathrm{umol} / \mathrm{ml} \mathrm{ChB}$ ), en cambio los tratamientos UVB100 y UVB150 (3.06 umol/ml ChA, $0.69 \mathrm{umol} / \mathrm{ml}$ ChB y $3.32 \mathrm{umol} / \mathrm{ml} \mathrm{ChA,} 1.07 \mathrm{umol} / \mathrm{ml} \mathrm{ChB}$ respectivamente) muestran disminución de la ChA en relación a la luz blanca (4.98 umol/ml ChA y $1.38 \mathrm{umol} / \mathrm{ml} \mathrm{ChB}$ ) (figura 4, tabla 8 y 9).

La disminución de clorofilas en los tratamientos UVB150 y UVB100 comparados con el control luz blanca y los otros tratamientos puede ser explicada debido a que las moléculas de clorofila son susceptibles a daños por fotoxidación causados por irradiaciones de alta intensidad como la UVB [42]. En cambio, bajas concentraciones de luz (UVA50) estimulan mecanismos para aumentar la densidad de pigmentos [43-44]. Además, la concentración de clorofilas parece no ser afectada en periodos cortos de irradiación, pero en exposición continua a radiaciones UVB100 y UVB150, como en nuestro diseño, causa una disminución de ChA - ChB, y puede estar relacionada a lesiones en los tilacoides [45].
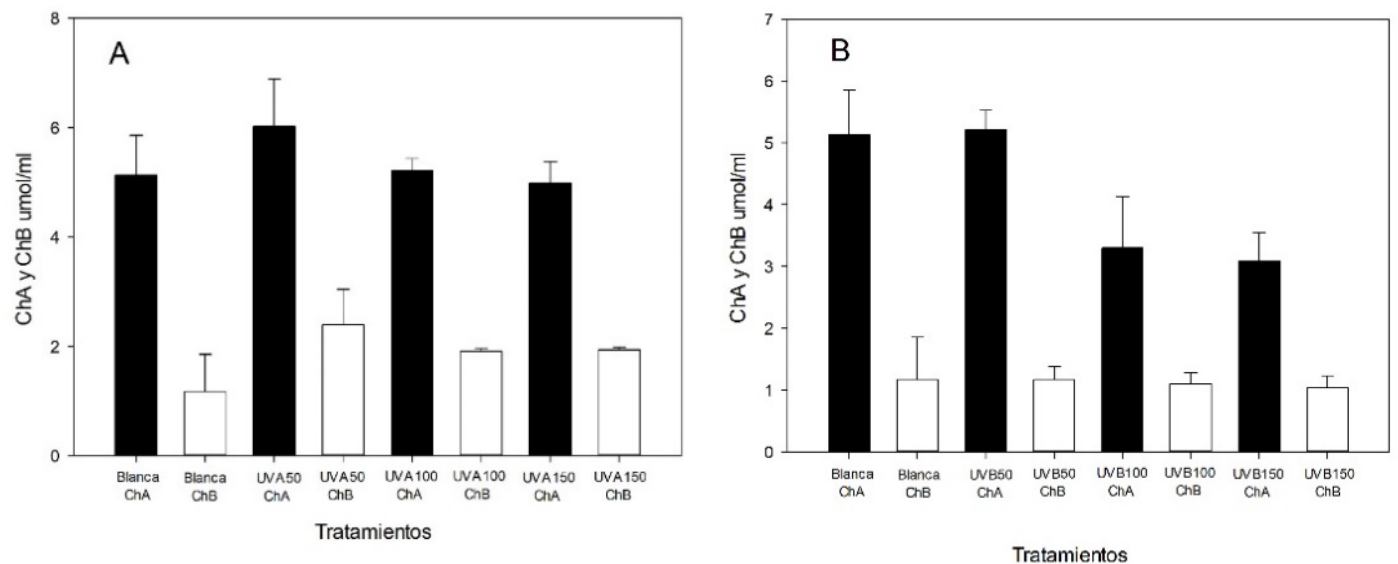

Figura 4. Concentración de ChA (A) y ChB (B) por extracción etanólica a las 8 semanas de los tratamientos A) UVA, B) UVB y control luz blanca. La ChA se muestra en columnas negras y la ChB en columnas blancas.

$\begin{array}{ccc}\begin{array}{c}\text { Downloadable from: Revista Boliviana } \\ \text { http://www.bolivianchemistryjournal.org, }\end{array} & 217 & \begin{array}{c}\text { Química. Volumen } 37 \text { N } 5 \text { 5. Año } 2020 \\ \text { http://www.scribd.com/bolivianjournalofchemistry }\end{array}\end{array}$




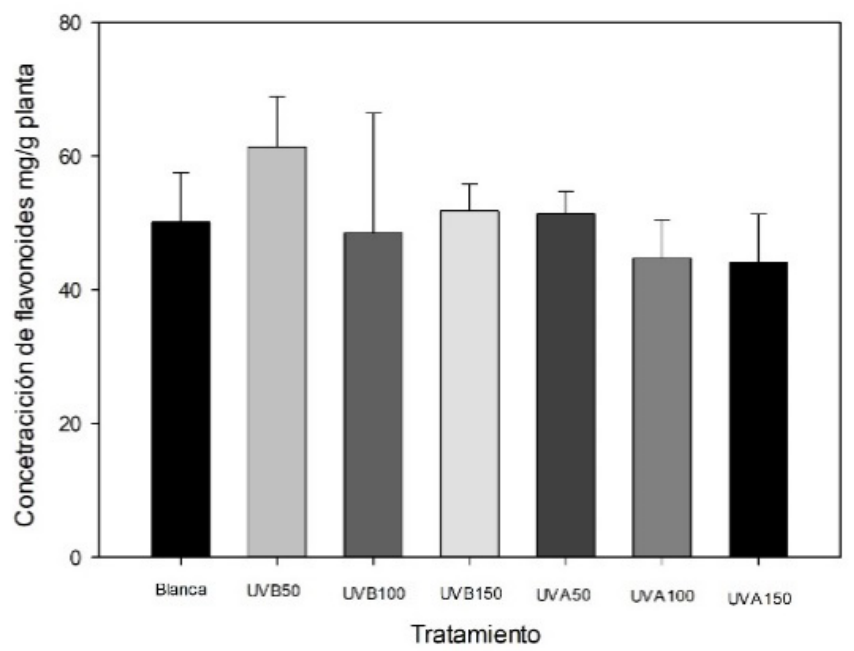

Figura 5. Concentración de flavonoides totales por extracción etanólica en relación a los tratamientos UVA, UVB y Blanca comparados a las 8 semanas.

Los resultados respecto a la concentración de flavonoides muestran diferencias significativas $(\mathrm{H}=2.73$, sig. $=0.04)$, se observa una mayor concentración en el tratamiento UVB50 (60.01 mg/g) en comparación de del control (46.13 $\mathrm{mg} / \mathrm{g}$ ), sugiriendo que bajos niveles de UVB favorecen a la concentración de flavonoides (figura 5, tabla 8 y 9), los demás tratamientos muestran resultados similares entre ellos (figura 5, tabla 9) siendo el tratamiento UVA150 el que presenta menor concentración $(42.42 \mathrm{mg} / \mathrm{g})$. Exposiciones prolongadas a altos niveles de luz pueden aumentar la concentración de flavonoides independientemente de las longitudes de onda [46-47]. Además, existe acumulación de flavonoides en relación al PAR, UVA o UVB diferenciadas, variar las longitudes de onda estimula diferentes flavonoides como por ejemplo el kaempferol es estimulado por el PAR y bajas dosis de UVA, en cambio la quercetina es estimulada por UVB y UVA, siendo así que la concentración de flavonoides totales puede ser similar, pero con diferentes composiciones [48-49]. Nuestros resultados también muestran que bajas dosis de UV pueden activar la síntesis flavonoides y acumularlos, en cambio dosis más altas de UV posiblemente pueden activar su utilización en la planta y reducir la concentración de los mismos, sin embargo, no muestran una tendencia lineal en UVB ( $\mathrm{y}=73.86$ $0.15 x ; R 2=0.67)$ o UVA ( $y=60.97-0.16 x ; R 2=0.79)$.

Respecto al análisis de variables conjuntas el tratamiento UVB50 muestra la mayor concentración de flavonoides, una favorable concentración de clorofilas y valores cercanos al control en la formación de tejidos foliares (figuras 4 y 5, tablas 7 y 9), sin embargo, muestra valores bajos respecto al crecimiento vegetativo (tabla 6 y 9). A demás de las diferencias entre el control y los tratamientos UV, ese observa que existen diferencias entre ambos tipos de longitud de onda UVA y UVB, la tabla 9 muestra que la UVA favorece positivamente a la concentración de clorofilas y las variables de anatomía foliar en comparación de la UVB. La UV induce cambios en la expresión genética, actividad enzimática, morfología y fisiología de la planta, además, la regulación de defensas antioxidantes en plantas puede ser detectada bajo muy baja radiación UV, promoviendo la formación de flavonoides a bajas concentraciones [15-47]. Asimismo, la disminución de pigmentos y el área foliar son respuesta identificadas en varias especies de plantas principalmente a radiación UVB de altas concentraciones [50-53] y pueden afectar a la fotosíntesis entre un 3 a $90 \%$ implicando otros efectos sobre la producción de otras moléculas al interferir el sistema de generación de energía de la planta [54].

Comparando todos los resultados obtenidos en este estudio se puede sugerir que las plantas de Baccharis latifolia presentarían mejores características de crecimiento y producción de compuestos químicos en bajos niveles de UV y valores altos de luz blanca. Estos resultados se pueden comparar con estudios realizados en campo [2-4], donde en época húmeda, existen $80 \%$ de días con radiación difusa (UV atenuada) y bajos valores de estrés ambiental principalmente relacionado a la humedad relativa y la humedad del suelo. La variación entre días nublados y despejados en época húmeda podría tener algún papel en el aumento de flavonoides reportado para la planta por otros estudios.

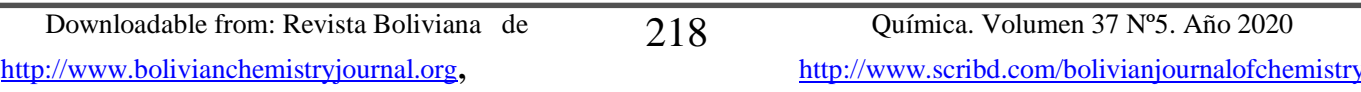


Tabla 8. Prueba Kruskal - Wallis en relación a las variables de respuesta fase 2, se observa diferencias significativas en las variables químicas Clorofila A (ChA) (<0.01), Clorofila B (ChB) (<0.01), Flavonoides totales (0.04); en las variables de crecimiento altura de la planta (<0.01), Número de hojas ( $<0.01)$; y variables anatómicas ancho de mesófilo $(<0.01)$, ancho de epidermis adaxial ( $<0.01)$, ancho de epidermis abaxial $(<0.01)$, parénquima en empalizada $(<0.01)$ y parénquima lagunar $(<0.01)$.

\begin{tabular}{l|lccr}
\multicolumn{1}{l}{ RESPUESTAS } & \multicolumn{1}{c}{ VARIABLE } & H DE KRUSKAL-WALLIS & GL & SIG. ASINTÓTICA \\
\hline QUÍMICAS & ChA & 29.75 & 6 & $<0.01^{*}$ \\
& ChB & 27.33 & 6 & $<0.01^{*}$ \\
& ChA/ChB & 23.98 & 6 & $<0.01^{*}$ \\
\cline { 2 - 3 } MORFOLÓGICAS & Flavonoides totales & 12.73 & 6 & $0.04^{*}$ \\
& Altura & 41.22 & 6 & $<0.01^{*}$ \\
& Número de hojas & 16.94 & 6 & $<0.01^{*}$ \\
& Biomasa & 0.32 & 6 & 0.99 \\
\multirow{3}{*}{ ANATÓMICAS } & Área Foliar & 9.43 & 6 & 0.15 \\
& Mesófilo & 37.47 & 6 & $<0.01^{*}$ \\
& E. adaxial & 26.02 & 6 & $<0.01^{*}$ \\
& E. abaxial & 22.63 & 6 & $<0.01^{*}$ \\
& PE & 36.43 & 6 & $<0.01^{*}$ \\
& PL & 31.53 & 6 & $<0.01^{*}$ \\
& Relación PE/PL & 7.81 & 6 & 0.25 \\
\hline
\end{tabular}

Tabla 9. Pruebas post-hoc Bonferroni en relación a las variables significativas de la fase 2 al 0.05 de nivel de confianza.

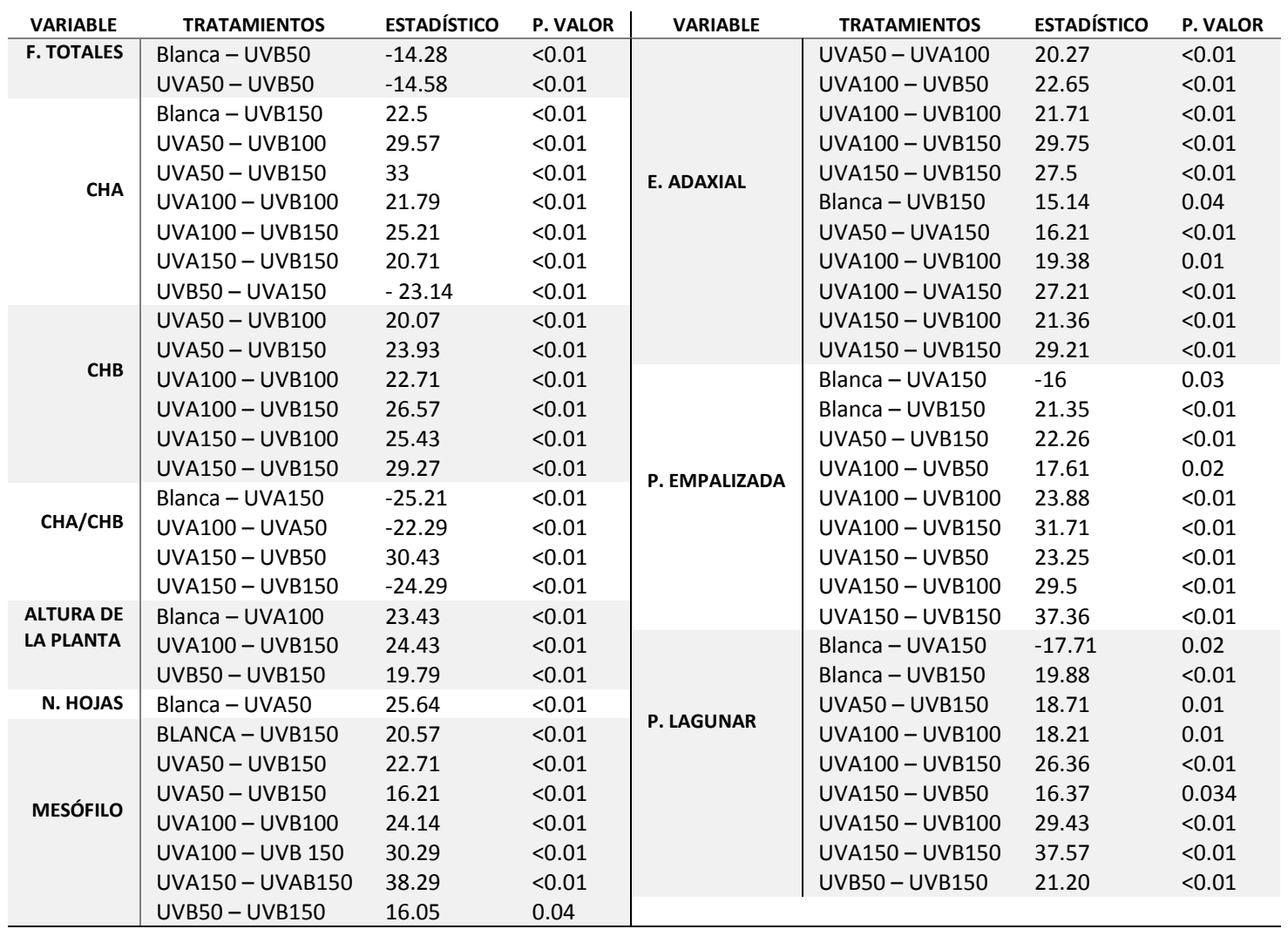

\section{CONCLUSIONES}

Baccharis latifolia es una especie plástica que puede acomodarse a varios niveles de luz y longitud de onda, sin embargo, presenta respuestas diferenciadas a nivel de su crecimiento, fisiología y anatomía foliar que repercuten en la concentración de compuestos fenólicos, clorofilas y crecimiento vegetativo. Niveles bajos de PAR reducen el crecimiento del tallo, la producción de flavonoides e incrementan la generación de hojas, concentración de clorofila y la formación de parénquima lagunar con espacios más amplios. La UVA favorece la formación biomasa, generación, Downloadable from: Revista Boliviana de http://www.bolivianchemistryjournal.org,
219 Química. Volumen 37 N5. Año 2020 http://www.scribd.com/bolivianjournalofchemistry 
elongación de hojas, mayor área foliar y aumento de los tejidos foliares, sin embargo, no incrementa la concentración de flavonoides. Por otro lado, la UVB a bajas concentraciones favorece la concentración de flavonoides, concentración de clorofila y formación de tejidos foliares, sin embargo, reduce el crecimiento vegetativo.

\section{RECONOCIMIENTOS}

Los autores expresan su agradecimiento a los Fondos para la Investigación IDH/UMSA por el financiamiento del proyecto: "Investigación de la influencia de variaciones meteorológicas y ecológicas en la producción de compuestos fitoterapéuticos de La Paz".

\section{REFERENCIAS}

1. Almanza, G.R., Salcedo, L. De la planta al medicamento. Parte I Investigaciones de Baccharis latifolia (Chilca), Editorial Académica Española, 1ra Edición, 2012, Saärbrucken, Alemania, 1-293.

2. Calle, A., San Martin, Á., Melgarejo, M., Flores, Y., Almanza, G. R. 2017, Evaluation of flavonoid contents and antibacterial activity of five bolivian Baccharis species, Revista Boliviana de Química., 34(4), 112-122.

3. San Martin, A. 2017, Identificación, cuantificación y propiedades farmacológicas de flavonoides de Baccharis latifolia (Chilca) y Arachis hypogaea (Maní), Tesis de Maestría en Ciencias Químicas, Universidad Mayor de San Andrés.

4. Enríquez, S., Quispe, R. E., Amurrio, P., Peñaranda, J. C., Calle, A., Orsag, V., Almanza, G. R. 2018, Contenidos flavonocidos en las hojas de Baccharis Latifolia, según el tipo de hoja, y su dependencia de las propiedades fisicoquímicas de los suelos. Revista Boliviana de Química, 35(5), 146-154.

5. Jagtap, V., Bhargava, S., Streb, P., Feierabend, J. 1998, Comparative effect of water, heat and light stresses on photosynthetic reactions in Sorghum bicolor (L.) Moench, Journal of Experimental Botany, 49(327), 1715-1721.

6. Long, S. P., Humphries, S., P., Falkowski, P. G. 1994, Photoinhibition of photosynthesis in nature. Annual review of plant biology, 45, 633-662.

7. Takahashi, S., Badger, M. R. 2011, Photoprotection in plants: a new light on photosystem II damage, Trends in plant science, 16(1), 5360 .

8. Lepistö, A., Rintamäki, E. 2012, Coordination of plastid and light signaling pathways upon development of Arabidopsis leaves under various photoperiods, Molecular plant, 5(4), 799-816.

9. Wilson, K. E., Thompson, J. E., Huner, N. P., B. M. Greenberg, B. M. 2001, Effects of Ultraviolet-A Exposure on Ultraviolet- B induced Accumulation of Specific Flavonoids in Brassica napus, Photochemistry and photobiology, 73(6), 678-684.

10. Osborne, B. A., G. T. Clabby, D. Horsley, \& Nolan, P. F. 1994, Is acclimation required for success in high light environments? A case study using Mycelis muralis (L.) Dumort (Asteraceae), New Phytologist, 127(2), 363-375.

11. Páez, A., Paz V., Páez. J. C. 2000, Crecimiento y respuestas fisiológicas de plantas de tomate cv. Río Grande en la época mayo-julio. Efecto del sombreado, Revista de la Facultad de Agronomía, 17, 173-184.

12. Kumari, R., Agrawal, S. B. 2010, Supplemental UV-B induced changes in leaf morpholog y, physiology and secondary metabolites of an Indian aromatic plant Cymbopogon citratus (DC) Staph under natural field conditions, International Journal of Environmental Studies, 67(5), 655-675.

13. Bornman, J., Barnes, F., Robinson, P. W., Ballare, S. A., Flint, C. L., Caldfwell, C. C. 2015, Solar ultraviolet radiation and ozone depletion-driven climate change: effects on terrestrial ecosystems, Photochemical \& Photobiological Sciences, 14(1), 88-107.

14. Boardman, H., Messier, C., Kneeshaw, D. D. 1999, Effects of light availability and sapling size on the growth, biomass allocation, and crown morphology of understory sugar maple, yellow birch, and beech, Ecoscience, 29, 345-356.

15. Robson, T. M., Aphalo, J. P., Banaś, A. K., Barnes, P. W., Brelsford, C. C., Jenkins, G. I., Kotilainen, T. K., Labuz, J., Martinez-Abaigar, J., Morales, L. O., Neugart, S., Pieriste, M., Rai, N., Vandenbussche, F., Jensen, M. A. 2019, A perspective on ecologically relevant plant-UV research and its practical application. Photochemical \& Photobiological Sciences, 18(5), 970-988.

16. Carvalho, N. O. S., Pelacani, C. R., Rodrigues, M. D., Crepaldi, I. C. 2006, Crescimento inicial de plantas de licuri (Syagrus coronata (Mart.) Becc.) em diferentes níveis de luminosidade, Revista Árvore, 30(3), 351-357.

17. Sumanta, N., Haque, C., Nishika, J., Suprakash, R. 2014, Spectrophotometric analysis of chlorophylls and carotenoids from commonly grown fern species by using various extracting solvents, Research Journal of Chemical Sciences, 4(9), 63-69

18. Baird, A. S., Anderegg, L. D., Lacey, M. E., Hilleris-Lambers, J., Van Volkenburgh, E. 2017, Comparative leaf growth strategies in response to low-water and low-light availability: variation in leaf physiology underlies variation in leaf mass per area in Populus tremuloides, Tree physiology, 37(9), 1140-1150.

19. Chang, C.C., Yang, M.H., Wen, H.M., Chern, J.C. 2002, Estimation of total flavonoid content in propolis by two complementary colorimetric methods, J. Food Drug Anal, 10(3), 178-182

20. Ramos, S. 2019, Contribución al estudio químico del género Baccharis de La Paz - Bolivia, Tesis de maestría en Ciencias Químicas, Universidad Mayor de San Andrés

21. Sartor, T., Xavier, V. B., Falcão, M. A., Mondin, C. A., Dos Santos, M. A., Cassel, E., Santarém, E. R. 2013, Seasonal changes in phenolic compounds and in the biological activities of Baccharis dentata (Vell.) GM Barroso. Industrial crops and products, 51, 355359.

22. Yoshida, S., Mandel, T., Kuhlemeier, C. 2011, Stem cell activation by light guides plant organogenesis, Genes \& Development, 25(13), 1439-1450.

Downloadable from: Revista Boliviana de
$220 \quad \begin{gathered}\text { Química. Volumen } 37 N^{\circ} 5 \text {. Año } 2020 \\ \text { http://www.scribd.com/bolivianjournalofchemistry }\end{gathered}$


23. Campos, N. R., Paciullo, D. S. C., Tavela, R. C., FreitasViana F. M., Bonaparte, T. P. 2007, Características Morfogênicas e Estruturais da Brachiaria de cumbens em Sistema Silviopastoril e Cultivo Exclusivo. Revista Brasileira de Biociências, 5(2):819- 821.

24. Lima, A. L. D. S., Zanella, F., \& Castro, L. D. M. D. 2010, Growth of Hymenae acourbaril L. var. stilbocarpa (Hayne) Lee et Lang. e Enterolobium contortisiliquum (Vell.) Morong (Leguminosae) under different shading levels, Acta amazonica, 40, 43-48.

25. Krause, G. H., Koroleva, O. Y., Dalling J. W., Winter, K. 2001, Acclimation of tropical tree seedlings to excessive light in simulated tree-fall gaps, Plant Cell \& Environment, 24(12), 1345-1352.

26. Barua, D., Heckathorn, S. A. 2006, The interactive effects of light and temperature on heat-shock protein accumulation in Solidago altissima (Asteraceae) in the field and laboratory, American Journal of Botany, 93, 102-109.

27. Bernal, M., Llorens, L., Julkunen-Tiitto, R., Badosa, J., Verdaguer. D. 2013, Altitudinal and seasonal changes of phenolic compounds in Buxus sempervirens leaves and cuticles, Plant Physiology Biochemistry, 70, 471-482.

28. Valladares, F., Niinemets, U. 2008, Shade tolerance, a key plant feature of complex nature and consequences, Annual Review of Ecology, Evolution, and Systematics, 39, 237-257.

29. Bidel, L. P., Meyer, S., Talhouët, A. C., Baudin, X., Daniel, C., Cazals, G., Streb, P. 2020, Epidermal UVA screening capacity measured in situ as an indicator of light acclimation state of leaves of a very plastic alpine plant Soldanella alpina L., Plant Physiology and Biochemistry, 151, 10-20.

30. Ghasemzadeh, A., Hawa, H., Jaafar, Z., Rahmat, A. 2010, Synthesis of phenolics and flavonoids in ginger (Zingiber officinale Roscoe) and their effects on photosynthesis rate, International Journal of Molecular Sciences, 11, 4539-4555.

31. Pacheco, F. V., Alvarenga, I. A. C., Junior, P. M. R., Pinto, J. E. B., Paula Avelar R., Alvarenga, A. A. 2014, Growth and production of secondary compounds in monkey-pepper (Piper aduncum L.) leaves cultivated under altered ambient light, Australian Journal of Crop Science, 8(11), 510-1516.

32. Páez, A., Paz, V., Páez, J. C. 2000, Crecimiento y respuestas fisiológicas de plantas de tomate cv. Río Grande en la época mayo-julio. Efecto del sombreado, Revista de la Facultad de Agronomía, 17, 173-184.

33. Lima, A. L. D. S., Zanella F., Castro, L. D. M. D. 2010, Growth of Hymenae acourbaril L. var. stilbocarpa (Hayne) Lee et Lang. e Enterolobium contortisiliquum (Vell.) Morong (Leguminosae) under different shading levels, Acta amazonica, 40, 43-48.

34. Neugart, S., Schreiner, M. 2018, UVB and UVA as eustressors in horticultural and agricultural crops, Scientia Horticulturae, 234, 370381.

35. Klem, K., Ač A., Holub, P., Kováč, D., Špunda, V., Robson T. M., Urban, O. 2012, Interactive effects of PAR and UV radiation on the physiology, morphology and leaf optical properties of two barley varieties, Environmental and experimental botany, 75, 52-64.

36. Kumari, S., Agrawal, M., Singh, A. 2015, Effects of ambient and elevated CO2 and ozone on physiological characteristics, antioxidative defense system and metabolites of potato in relation to ozone flux, Environmental and experimental botany, $109,276-287$.

37. Sakalauskaite, J., Viskelis, P., Dambrauskienė, E., Sakalauskienė, S., Samuolienė, G., Brazaitytė, Urbonavičienė, D. 2013, The effects of different UV -B radiation intensities on morphological and biochemical characteristics in Ocimum basilicum L., Journal of the Science of Food and Agriculture, 93(6), 1266-1271.

38. De Araújo, R. P., de Almeida, A. A. F., Barroso, J. P., de Oliveira, R. A., Gomes, F. P., Ahnert, D., Baligar, V. 2017, Molecular and morphophysiological responses cocoa leaves with different concentrations of anthocyanin to variations in light levels, Scientia Horticulturae, 224, 188-197.

39. Wyka, T., Robakowsky, P., Ytkowiak, R. 2007, Acclimation of leaves to contrasting irradiance in juvenile trees differing in shade tolerance, Tree Physiol, 27, 1293-1306.

40. Kakani V. G., Reddy K. R., Zhao D., Mohammed A. R. 2003, Effects of ultraviolet-B radiation on cotton (Gossypium hirsutum L.) morphology and anatomy, Annals of Botany, 91, 817-826.

41. Kakani V. G., Reddy K. R., Zhao D., Gao W. 2004, Senescence and hyperspectral reflectance of cotton levels exposed to ultraviolet radiation and carbon dioxide, Physiologia. Plantarum, 121, 250-257.

42. Wongsen, W., Bodhipadma, K., Noichinda, S., Leung, D. 2013, Relationship between leaf position and antioxidant properties in three basil species. International Food Research Journal, I20(3), 1113.

43. Alvarenga, A. A. D., Castro, E. M. D., Junior, L., de Castro, É., Magalhães, M. 2003, Effects of different light levels on the initial growth and photosynthesis of Croton urucurana Baill. in southeastern Brazil, Revista Árvore, 27(1), 53-57.

44. Bidel, L. P. R., Meyer, S., Goulas, Y., Cadot, Y., Cerovic, Z. 2007, Responses of epidermal phenolic compounds to light acclimation: in vivo qualitative and quantitative assessment using chlorophyll fluorescence excitation spectra in leaves of three woody species, Journal of Photochemistry and Photobiology B: Biology, 88(2-3), 163-179.

45. Juozaitytė, R., Ramaškevičienè, A., Sliesaravičius, A., Burbulis, N., Kuprienè, R., Liakas, V., \& Blinstrubienė, A. 2008, Effects of UVB radiation on photosynthesis pigment system and growth of pea (Pisum sativum L.). Works Lithuanian Inst. Hortic. Lithuanian Univ. Agric, 27, 179-186.

46. Fini, A., Brunetti, C., Di Ferdinando, M., Ferrini, F., Tattini, M. 2011, Stress-induced flavonoid biosynthesis and the antioxidant machinery of plants, Plant Signal. Behav, 6, 709-711.

47. Tattini, M., Landi, M., Brunetti, C., Giordano, C., Remorini, D., Gould, KS, Guidi, L. 2014, Las antocianinas de cumeroil epidérmicas protegen la albahaca dulce contra el exceso de estrés lumínico: múltiples consecuencias de la atenuación de la luz, Physiologia plantarum, 152(3), 585-598.

48. Wilson, K. E., Thompson, J. E., Huner, N. P., Greenberg, B. M. 2001. Effects of Ultraviolet-A Exposure on Ultraviolet- B -induced Accumulation of Specific Flavonoids in Brassica napus, Photochemistry and photobiology, 73(6), 678-684.

49. Verdaguer, D., Llorens, L., Bernal, M., Badosa, J. 2012. Photomorphogenic effects of UVB and UVA radiation on leaves of six Mediterranean sclerophyllous woody species subjected to two different watering regimes at the seedling stage, Environmental and experimental botany, 79, 66-75.

$\begin{aligned} & \text { Downloadable from: Revista Boliviana de } \\ & \text { http://www.bolivianchemistryjournal.org, }\end{aligned}$
$\underline{\underline{y}}$


ISSN 2078-3949 Rev, boliv. quim. Electronic edition

Published 1230 2020; DOI:10.34098/2078-3949.37.5.1

Juan C. Peñaranda et al. RBQ Vol.37, No.5, pp. 210-222, 2020

50. Surabhi, G. K., Reddy, K. R., Singh, S. K. 2009, Photosynthesis, fluorescence, shoot biomass and seed weight responses of three cowpea (Vigna unguiculata (L.) Walp.) cultivars with contrasting sensitivity to UV-B radiation, Environ. Exp. Bot., 66, 160-171.

51. Tang, W. T., Liu, X., Fang, M. F., Yue, M. 2011, FTIR Analysis of the Effects of Enhanced Ultraviolet-B (UV-B) Radiation on Chemical Composition of Different Parts of Scutellaria baicalensis Georgi, Spectrosc. Spectr, Anal, 31, 1220-1224.

52. Quan, J., Song, S., Abdulrashid, K., Chai, Y., Yue, M., Liu, X. 2018, Separate and combined response to UV-B radiation and jasmonic acid on photosynthesis and growth characteristics of Scutellaria baicalensis, International journal of molecular sciences, $19(4), 1194$.

Downloadable from: Revista Boliviana de http://www.bolivianchemistryjournal.org,
222

Química. Volumen 37 N5. Año 2020

http://www.scribd.com/bolivianjournalofchemistry 